\title{
Optimal Distributed Generation and Reactive Power Allocation in Electrical Distribution Systems
}

\author{
Benvindo R. Pereira Jr., Geraldo R. Martins da Costa, Member, IEEE, Javier Contreras, Fellow, IEEE, \\ and José R. Sanches Mantovani, Member, IEEE
}

\begin{abstract}
Optimal and simultaneous siting and sizing of distributed generators and capacitor banks in distribution systems have attracted a lot of attention from distribution companies. The placement and capacity of these devices have direct effects on the system's performance. This paper presents a model for the simultaneous allocation of capacitor banks and distributed generation, which takes into account the stochastic nature of distributed generation. To solve the model presented, we propose an efficient hybrid method based on Tabu search and genetic algorithms. The hybrid method is applied to a well-known system in literature.
\end{abstract}

Index Terms-Capacitor bank (CB), distributed generation (DG), uncertainty, Tabu search (TB), Chu-Beasley genetic algorithm (CBGA).

\section{NOTATION}

Sets

$n b, n b c, n b g$

Sets of system buses, candidate buses for CB installation, and candidate buses for DG installation.

nlo Set of load levels.

$n l \quad$ Set of system lines.

$C B_{f}, C B_{s} \quad$ Set of fixed and switched CBs available to be installed.

$D G \quad$ Set of DG units available to be installed.

Costs

$C B c_{f c}^{c}, C B c_{s c}^{c} \quad$ Cost to install a fixed/switched CB of type $c$, in $\$$.

$D G c^{g}$

Cost to install a DG unit of type $g$, in $\$$.

$K s_{l}$

Substation operating cost with load level $l$, in $\$ / M W h$.

$K g_{g, l}$ Operating cost of DG type $g$ with load level $l$, in $\$$ /MVAh.

Manuscript received August 13, 2015; revised November 06, 2015; accepted December 17, 2015. Date of publication January 20, 2016; date of current version June 16, 2016. This work was supported by FAPESP under Grants 2013/23124-7 and 2015/15605-0. Paper no. TSTE-00678-2015.

B. R. Pereira Jr. and G. R. M. da Costa are with the Department of Electrical Engineering and Computational, University of São Paulo, 13162-150 São Carlos, Brazil (e-mail: brpjunior@gmail.com; geraldo@sc.usp.br).

J. Contreras is with E.T.S. de Ingenieros Industriales, University of CastillaLa Mancha, 13071 Ciudad Real, Spain (e-mail: Javier.Contreras@uclm.es).

J. R. S. Mantovani is with the Department of Electrical Engineering, São Paulo State University, 15385-000 Ilha Solteira, Brazil (e-mail: mant@dee.feis.unesp.br).

Color versions of one or more of the figures in this paper are available online at http://ieeexplore.ieee.org.

Digital Object Identifier 10.1109/TSTE.2015.2512819

\section{Binary Variables}

$x_{f c}^{c, i}, x_{s c}^{c, i} \quad$ Decision to install (1) or not (0) a fixed/switched capacitor bank of type $c$ at candidate bus $i$.
$x_{D G}^{g, i}$
Decision to install (1) or not (0) a DG unit of type $g$ at candidate bus $i$.
$x_{o s c}^{c, i, l}$
Decision to operate (1) or not (0) a switched CB of type $c$ installed at candidate bus $i$, with load level $l$.

\section{Continuous Variables}

$I_{i j, l, p} \quad$ Current through branch $i j$ with load level $l$, $P_{D G}^{g, i, l}, Q_{D G}^{g, i, l}$ in $\mathrm{A}$.

Active and reactive power generated by a DG unit of type $g$ installed in the candidate bus $l$ with load level $l$, in MW and MVAr.

$x_{o s c}^{c, i, l}$

Decision to operate (1) or not (0) a switched CB of type $c$ installed at candidate bus $i$, with load level $l$.

$P_{i}^{l}(V, \theta), Q_{i}^{l}(V, \theta)$ Active and reactive power injection at bus $i$ with load level $l$, in MW and MVAr.

$P G_{i}^{l}, Q G_{i}^{l} \quad$ Active and reactive power generated at bus $i$ with load level $l$, in MW and MVAr.

$P_{l}^{S, l, p}$ Voltage at bus $i$ with load level $l$, in V. Active power injected in the system through the substation with load level $l$, in MW.

$S S \_p f_{l}$ Power factor measured at the substation with load level $l$.

\section{Parameters}

$\alpha^{\text {ope }}$

$\varphi_{c} \quad$ Angle of the maximum capacitive power factor.

$\varphi_{i} \quad$ Angle of the maximum inductive power

$I_{i j}^{\max }$ factor.

Maximum current allowed through branch $P_{D G \max }^{g}, P_{D G \min }^{g}$ Maximum and minimum active power lim$P L_{i}^{l}, Q L_{i}^{l}$ its of a DG unit of type $g$, in MW.

with load level $l$, in MW and MVAr.

$Q C_{f c}^{c}, Q C_{s c}^{c} \quad$ Reactive power of a fixed/switched CB of

$Q C_{\max } \quad$ Maximum reactive power injected by a CB type $c$, in $\mathrm{kVAr}$. $Q_{D G \max }^{g}\left(P_{D G}^{g, i, l}\right)$, Maximum and minimum reactive power $Q_{D G \min }^{g}\left(P_{D G}^{g, i, l}\right) \quad$ limit for a DG unit of type $g$, in MVAr. 


$\begin{array}{ll}S S_{\_} p f_{\max }, & \text { Maximum and minimum power factors } \\ S S_{\_} p f_{\min } & \text { acceptable at the substation. } \\ S_{D G \max }^{g} & \begin{array}{l}\text { Maximum apparent power of a DG unit of } \\ \text { type } g, \text { in MVA. }\end{array} \\ T_{l} & \begin{array}{l}\text { Duration of load level } l, \text { in hours. } \\ V_{\max }, V_{\min }\end{array} \\ \begin{array}{l}\text { Maximum and minimum voltage levels of } \\ \text { the system, in V. }\end{array}\end{array}$

\section{INTRODUCTION}

$\mathbf{T}$ HE INSTALLATION of distributed generation (DG) in medium- and low-voltage distribution systems has become a feasible option in distribution system planning. DG can offer several benefits to the system, however, its installation is subject to the availability and geographical location of primary energy sources. The benefits obtained with DG are not only related to the technical level (loss reduction, voltage control, current flow reduction in the branches, improved quality of power supply [1]), but also to the environmental sphere, since the reduction of costs and technological advances in power electronics, communication systems, control and automation allow for the use of renewable energy sources which pollute less than conventional generators.

On the other hand, DG can cause undesired effects in the system, such as fluctuations in the voltage profile, increased fault current, inversion in the flow direction, readjustment of the control and protection systems, etc. [1]. These effects become more evident when DG uses renewable energy sources, since, in their great majority, they have a probabilistic nature, such as wind speed and solar irradiation. Therefore, technical studies should be conducted so that DG can be properly installed in passive systems, avoiding the degradation of reliability, system operation, and quality of supply [2]. The need for such studies has, in the recent past, promoted research on DG regarding topics related to its operating mode [3] and the operation and expansion of distribution systems [1], [2], [4], [5].

In passive distribution systems, some of the benefits achieved by the presence of DG are assigned to other devices, among which capacitor banks (CBs) stand out. CB are devices widely used due to their efficiency in reactive compensation, and, when optimally installed in distribution systems, provide loss reduction, voltage regulation, and a financial return for distribution companies (DISCOs) [6]-[10].

In active distribution systems the benefits and undesired effects obtained by the installation or existence of DG and CB are directly related to the location of these devices in the network. The task of finding the location of these devices where the benefits are maximized is not a simple endeavor, since it is a combinatorial problem and involves the development of a complex mathematical model composed of continuous, binary, and integer variables. Different models and solution techniques are found in literature for the CB allocation problem [6]-[10] and the DG allocation problem [1], [5]. Considering the reality of modern distribution systems, in which DG and CB must coexist, it is necessary to review the allocation techniques, taking into account the simultaneous allocation of CB and DG in the system, once $\mathrm{CB}$ and DG provide similar and complementary benefits to distribution systems [2], [11]-[14].
This paper presents a new methodology for the optimal and simultaneous allocation of DG and CB in distribution systems, differing from the proposals found in literature in the way different types of DG are modeled in the problem, in the objective function, and in the CB type. Thus, the main contributions of this paper are as follows:

- Simultaneous allocation of (fixed and switched) CBs and DG (dispatchable and stochastic) in the distribution systems is presented;

- In addition to dispatchable DG modeled using synchronous generators (SGs), the proposed model considers stochastic DG represented by wind turbines modeled as doubly-fed induction generators (DFIGs) [3], [15];

- A probabilistic approach to find the power dispatch of stochastic DG is used in order to achieve a more realistic representation of the impacts of DG on distribution systems and, consequently, in the simultaneous allocation of CBs and DG;

- Different load levels (to represent a daily load curve) are taken into account in the model to find the operational control of swicthed CBs;

To solve the proposed model we have used a hybrid algorithm composed of a Tabu Search (TS) algorithm and a Chu-Beasley Genetic Algorithm (CBGA). In addition to being powerful tools that solve large and complex mixed nonlinear combinatorial problems [10], [12], [14], [16], these metaheuristics enable us to represent the sitting, sizing and control scheme of DG and CBs in a easy and practical way.

TS is the main algorithm responsible for finding: location of $\mathrm{CB}$, location of DG, and type of DG. The CBGA is used to solve an optimal power flow (OPF), which is an important tool responsible for finding: DG power dispatch (dispatchable and stochastic), type of $\mathrm{CB}$, and the operational scheme of $\mathrm{CB}$, considering the probabilistic nature of DG sources. In the proposed model the objective function aims to minimize investment costs (installation of DG and $\mathrm{CB}$ ) and system operation costs (power generation by DG and power purchase through the substation), subject to technical and operational constraints, as well as the availability of DG power generation. The results obtained allow us to perform a specific analysis of the system's behavior.

The remaining parts of the paper are divided as follows. Section II presents the mathematical formulation, Section III describes the proposed methodology, Section IV presents the results for a well-known system in literature and several conclusions are presented in Section V.

\section{Mathematical Model}

The problem of the simultaneous allocation of CB and DG is modeled as a mixed-integer non-linear problem. In the formulation of this model we have considered the following hypotheses for DG and CB:

- DG belongs to DISCOs [2], [12], [17], [18];

- Dispatchable DG: full control of the active and reactive power generated is possible.

- Stochastic DG injects all its active power production into the system and the control over reactive power is done by controlling the power factor. 
- DG can produce reactive power only if producing active power;

Note that the first hypothesis could be related to the role of DISCOS as market facilitators as mentioned in [19]. The mathematical model proposed for the optimal CB and DG allocation is as follows:

$$
\begin{aligned}
\text { Of }= & \min \left(I n v_{\text {costs }}+O p e_{\text {costs }}\right) \\
\text { Inv }_{\text {costs }}= & \sum_{i \in n b c}\left(\sum_{c \in C B_{f}} C B c_{f c}^{c} \cdot x_{f c}^{c, i}+\sum_{c \in C B_{s}} C B c_{s c}^{c} \cdot x_{s c}^{c, i}\right) \\
& +\sum_{i \in n b g} \sum_{g \in D G} D G c^{g} \cdot x_{D G}^{g, i} \\
O p e_{\text {costs }}= & \sum_{l \in n l o}\left(P_{l}^{S S} \cdot T_{l} \cdot K s_{l}\right. \\
& \left.+\sum_{i \in n b g} \sum_{g \in D G} \sqrt{P_{D G}^{g, i, l^{2}}+Q_{D G}^{g, i, l^{2}}} \cdot T_{l} \cdot K g_{g, l}\right) \cdot \alpha^{o p e}
\end{aligned}
$$

subject to:

$$
\begin{aligned}
& P G_{i}^{l}-P L_{i}^{l}-P_{i}^{l}(\theta, V)=0 \quad \forall: i \in n b, l \in \text { nlo } \\
& Q G_{i}^{l}+\sum_{c \in C B_{f}} Q C_{f c}^{c} \cdot x_{f c}^{c, i}+\sum_{c \in C B_{s}} Q C_{s c}^{c} \cdot x_{o s c}^{c, i, l} \\
& -Q L_{i}^{l}-Q_{i}^{l}(\theta, V)=0 \quad \forall: i \in n b, l \in \text { nlo } \\
& \sum_{c \in C B_{f}} Q C_{f c}^{c} \cdot x_{f c}^{c, i}+\sum_{c \in C B_{s}} Q C_{s c}^{c} \cdot x_{o s c}^{c, i, l} \leq Q C^{\max } \\
& \forall: i \in n b, l \in \text { nlo } \\
& P G_{i}^{l}=\sum_{g \in D G} P_{D G}^{g, i, l} \quad \forall: i \in n b g, l \in \text { nlo } \\
& Q G_{i}^{l}=\sum_{g \in D G} Q_{D G}^{g, i, l} \quad \forall: i \in n b g, l \in \text { nlo } \\
& x_{D G}^{g, i} \cdot P_{D G_{\text {min }}}^{g} \leq P_{D G}^{g, i, l} \leq P_{D G_{\text {max }}}^{g} \cdot x_{D G}^{g i} \\
& \forall: i \in n b g, g \in D G, l \in \text { nlo } \\
& x_{D G}^{g, i} \cdot Q_{D G_{\text {min }}}^{g}\left(P_{D G}^{g, i, l}\right) \leq Q_{D G}^{g, i, l} \leq Q_{D G_{\text {max }}}^{g}\left(P_{D G}^{g, i, l}\right) \cdot x_{D G}^{g, i} \\
& \forall: i \in n b g, g \in D G, l \in \text { nlo } \\
& \sqrt{P_{D G}^{g, i, l^{2}}+Q_{D G}^{g, i, l^{2}}} \leq x_{D G}^{g, n} \cdot S_{D G_{\max }}^{g} \\
& \forall: i \in n b g, g \in D G, l \in \text { nlo } \\
& P_{D G}^{g, i, l} \geq 0 \quad \forall: i \in n b g, g \in D G, l \in \text { nlo } \\
& S S \_p f_{\text {min }} \leq S S \_p f_{l} \leq S S \_p f_{\text {max }} \quad \forall: l \in \text { nlo } \\
& V_{\text {min }} \leq V_{i, l} \leq V_{\text {max }} \quad \forall: i \in n b, l \in \text { nlo } \\
& I_{i j, l} \leq I_{i j}^{\max } \quad \forall: i j \in n l, l \in \text { nlo } \\
& x_{o s c}^{c, i, l} \leq x_{s c}^{c, i} \quad \forall: i j \in n l, l \in n l o \\
& \sum_{g \in D G} x_{D G}^{g, n} \leq 1 \quad \forall: i \in n b g \\
& x_{f c}^{c, i}, x_{s c}^{c, i}, x_{o s c}^{c, i, l}, x_{D G}^{g, i} \in\{0,1\}
\end{aligned}
$$

The objective function aims to minimize investment (2) and operational (3) costs. Operational costs take into account the
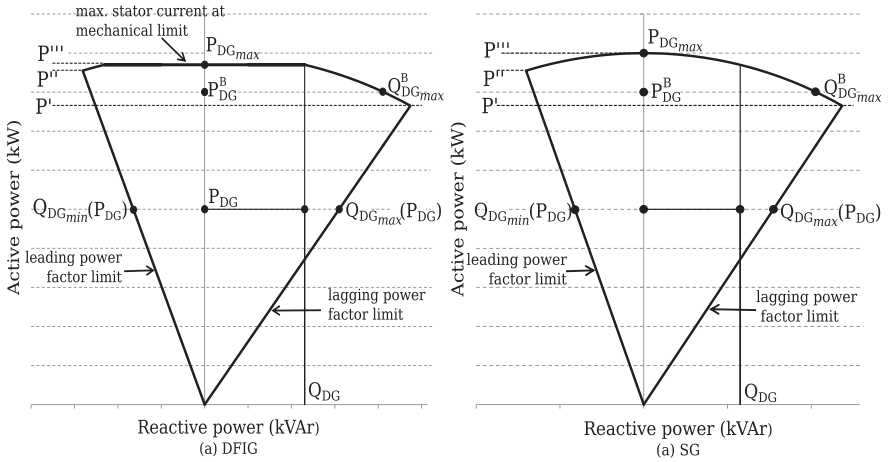

Fig. 1. Operational region of DFIGs and SGs.

different expenses involved in energy acquisition, such as the purchase of energy through the substation and power production by DG, including reactive power injection by DG. The constraints considered in the problem are those traditionally used in literature [7]-[10], [12], [16]: power flow balance $(4,5)$, maximum reactive power injection by $\mathrm{CB}$ at a specific bus (6), reverse active power flow at substation (12), power factor limits at the substation (13), voltage limits in the system's buses (14), current limits in the system's lines (15), and switched CB operation only if it is already installed (16), and constraint (17) ensures that only one type of DG is installed at a specific candidate bus.

In addition to these constraints, due to the presence of DG, it is necessary to add the physical and operational constraints of these devices. In the mathematical model this is achieved through (9), (10), and (11), limiting DG power production. These equations are subject to the generator types and their capability curves. In this paper, attention is focused on two generator types: doubly-fed induction generators (DFIGs) [3], [15], more common in wind energy applications, and synchronous generators (SGs) to represent dispatchable generation, although its application is possible in wind turbines. Considering (9), (10), and (11), DG hypotheses, and DFIG capability curves, the operational region for these types of DG are presented in Fig. 1. For DFIGs, the limits of $Q_{D G}^{g, n, l}$ in (10) are defined by the following equations:

- if $0 \leq P_{D G}^{g, n, l} \leq P^{\prime}$ :

$$
-P_{D G}^{g, n, l} \cdot \tan \varphi_{c} \leq Q_{D G}^{g, n, l} \leq P_{D G}^{g, n, l} \cdot \tan \varphi_{i}
$$

- if $P^{\prime} \leq P_{D G}^{g, n, l} \leq P^{\prime \prime}$ :

$$
-P_{D G}^{g, n, l} \cdot \tan \varphi_{c} \leq Q_{D G}^{g, n, l} \leq \sqrt{S_{D G_{\max }}^{g}-P_{D G}^{g, n, l^{2}}}
$$

- if $P^{\prime \prime} \leq P_{D G}^{g, n, l} \leq P^{\prime \prime \prime}$ :

$$
\begin{gathered}
-\sqrt{S_{D G_{\max }{ }^{2}-P_{D G}^{g, n, l^{2}}}^{g}} \leq Q_{D G}^{g, n, l} \\
\leq \sqrt{S_{D G_{\max }{ }^{2}-P_{D G}^{g, n, l^{2}}}^{g}}
\end{gathered}
$$

- if $P_{D G}^{g, n, l}=P^{\prime \prime \prime}$ :

$$
\begin{aligned}
& -\sqrt{{S_{D G_{\max }}^{g}-P_{D G_{\max }}^{g}}^{2}} \leq Q_{D G}^{g, n, l} \\
& \leq \sqrt{S_{D G_{\max }}^{g}{ }^{2}-P_{D G_{\max }}^{g}{ }^{2}}
\end{aligned}
$$




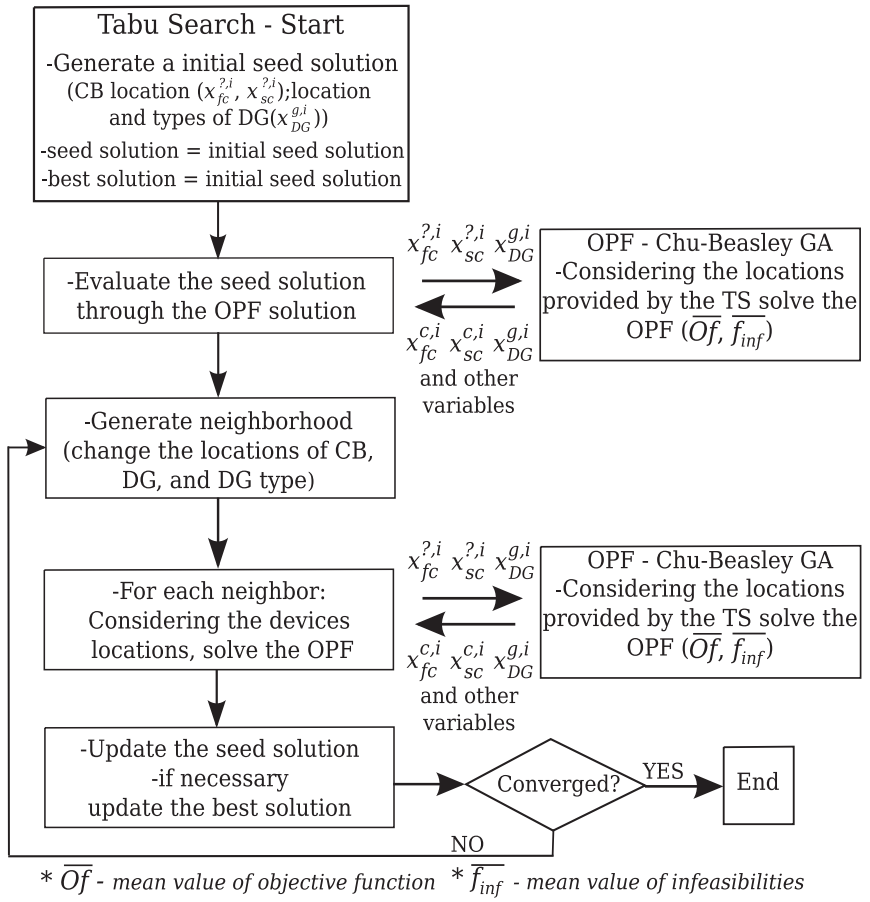

Fig. 2. Diagram of the proposed methodology.

For SGs, the limits of $Q_{D G}^{g, n, l}$ in (10) are defined by (19), (20), (21).

\section{Proposed Methodology}

To solve the model presented in Section II we have used a hybrid algorithm composed of a Tabu Search (TS) algorithm [20] and a Chu-Beasley Genetic Algorithm (CBGA) [21].

TS is used to find the position of CB and DG in the system, as well as the DG types. Its efficiency is subject to two important concepts: codification and neighborhood. In the codification a decimal base is used to represent in an easy and practical way the devices' positions [22] in the system and the DG types. The neighborhood structure consists of amending the position of a $\mathrm{CB}$, DG or the DG type. In this case, a neighbor differs from the current solution in only one position or DG type.

Knowing the devices' locations in the network and the types of DG, an optimal power flow (OPF) is solved to find:

- the types of CB to be allocated;

- the dispatch of active and reactive power (through operational power factor) of dispatchable DG;

- the operational power factor (for reactive power production) of stochastic DG.

To solve the OPF model we have used a CBGA detailed in subsection III-B and the uncertainties of the stochastic DG are considered in the OPF solution process through the $2 m+1$ point estimate method (PEM), a well-known method in literature [23]. A block diagram of the proposed methodology is presented in Fig. 2.

\section{A. $2 m+1$ PEM Applied to the Optimal Allocation Problem of $C B$ and $D G$}

The development of new technologies has made it possible for many forms of energy to be used in electricity production, highlighting clean energies, such as solar and wind power. These energy sources have a high degree of uncertainty, requiring the use of appropriate techniques to assess their impact on the power system.

Monte Carlo simulation is a powerful tool that has been widely used for this type of analysis, however it requires a large number of trial runs and the knowledge of the probability distribution function (PDF) of the random variable [24]. To work around these problems of Monte Carlo simulation (MCS), $2 m+1$ PEM [23], [25] is used in the DG and CB allocation problem.

PEM uses statistical information of the input random variables belonging to the problem and function $F$, which relates input and output variables, to find information about the output variables' uncertainty. The estimated $K$ points (concentrations), 2 in this case, are composed of two parts: location $\left(p_{l, k}\right)$ and weight $\left(w_{l, k}\right)$. The location $p_{l, k}$ is the $k$-th value of the variable at which the function is evaluated and $w_{l, k}$ is a weighting factor which accounts for the relative importance of this evaluation in the output random variables [23], [25]. Thus, for each random variable, $K$ deterministic problems need to be solved considering each $p_{l, k}$ and other random variables are fixed at their corresponding mean values $\left(\mu_{p 1}, \mu_{p 2}, \ldots, p_{l, k}, \ldots \mu_{p m}\right)$ [23], [25]. In addition to these points one more evaluation of function $F$ is required, and this concentration consists of $m$ input random variables at their mean values $\left(\mu_{p 1}, \mu_{p 2}, \ldots, \mu_{p l}, \ldots \mu_{p m}\right)$ [23], [25].

For each concentration $\left(\mu_{p 1}, \mu_{p 2}, \ldots, p_{l, k}, \ldots \mu_{p m}\right)$ a vector of output variables, $Z(l, k)$, is obtained by evaluating $F$. By using $w_{l, k}$ it is possible to find the $j$-th moment of the output variables. For example, the first and second moments, mean and variance, can be found by using the following equations:

$$
\begin{aligned}
\mu_{Z} & =E[Z] \cong \sum_{l=1}^{m} \sum_{k=1}^{2} \mu_{l, k} \cdot(Z(l, k)) \\
\operatorname{Var}_{Z} & =E\left[Z^{2}\right] \cong \sum_{l=1}^{m} \sum_{k=1}^{2} \mu_{l, k} \cdot(Z(l, k))^{2} \\
\sigma_{Z} & =\sqrt{\operatorname{Var}_{Z}}=\sqrt{E\left[Z^{2}\right]-E[Z]^{2}}
\end{aligned}
$$

In the optimal and simultaneous allocation of CB and DG, function $F$ comes from the model presented in eq. (1-18) and this deterministic model is evaluated through a backward/ forward power flow solution [26]. The input random variables are the active power injection of the stochastic DG, calculated through the wind speed, and the output variables are costs, infeasibilities, and all the variables related to the power flow.

\section{B. Optimal Power Flow (OPF)}

To evaluate each solution provided by TS, an OPF is solved to find the active and reactive DG power injections and the $\mathrm{CB}$ types, as well as their operational schemes for each load level. If there are stochastic DG units installed in the system, $2 m+1$ PEM is used to properly consider their active power injections, changing the deterministic value $O f(1)$ to a stochastic value with mean value $\overline{O f}$. In this paper reactive power control of DG is achieved through power factor (PF) control, due 
to the voltage variation caused by stochastic DG. This consideration provides a more efficient control of DG reactive power injection, since it does not depend on voltage.

The OPF model solved is the one presented in (1-18) fixing the $i$ indexes of variables $x_{f c}^{c, i}, x_{s c}^{c, i}$ and $x_{D G}^{g, i}$ as specified by each solution of the TS algorithm. To solve the OPF model a Genetic Algorithm (GA) is used [27], [21]. The GA developed for the OPF solution is shown in algorithm 1.

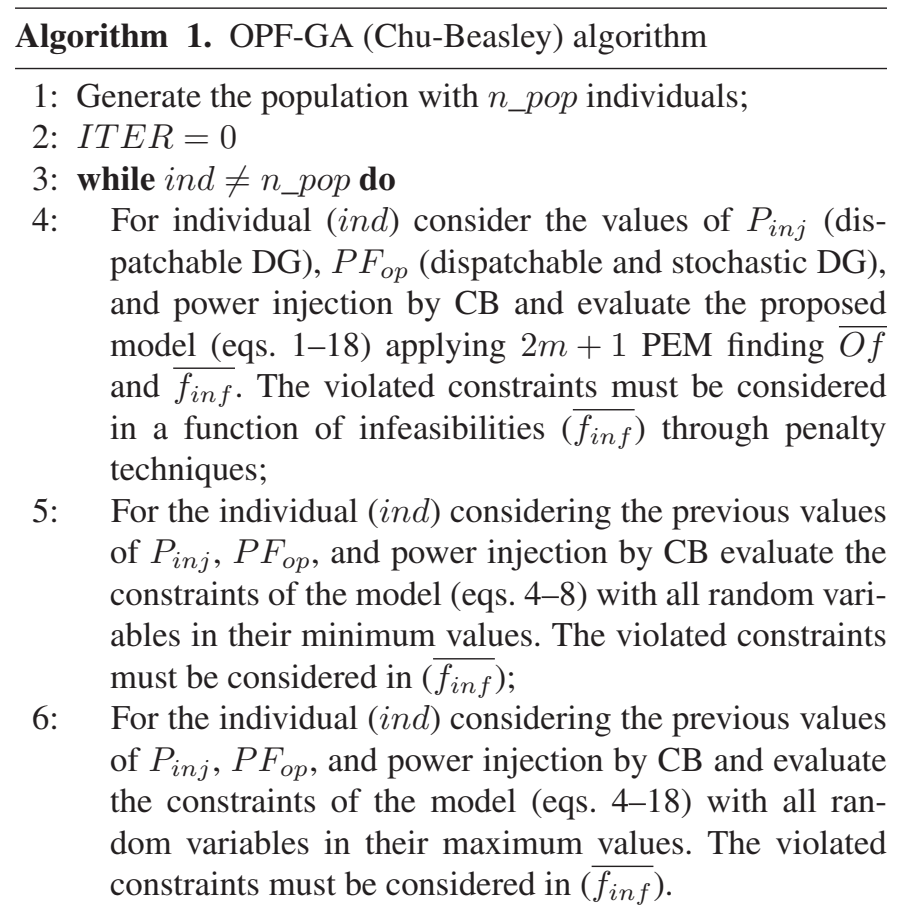

\section{7: end while}

8: $c_{\text {iter }}=0$;

9: while $I T E R \leq I T E R_{\max }$ do

10: $\quad I T E R=I T E R+1$;

11: Select two individuals of the population through the selection tournament process;

12: Apply the crossover and mutation operators;

13: For the new individual (nind) generated, find $\left(\overline{O f}^{\text {nind }}\right.$ ) and $\overline{f_{\text {inf }}}$ nind

14: if (The new individual is different from all individuals in the population = "yes") then

15: if $\left(\overline{f_{\text {inf }}}\right.$ of all the individuals in the population is zero $=$ "yes") then

16: $\quad$ Find the individual with the worst value of $\overline{O f}$ in the population (wind);

$17:$

$18:$

19:

20:

21:

22:

23:

24:

25:

26:
27

\section{8:}

29:

30:

31:

32:

33:

34:

35:

\section{end if}

36: end while

37: end

\section{Evaluation of Two New Points in the OPF}

In addition to the $2 m+1$ points analyzed in the PEM, this paper proposes two new points to be evaluated in the OPF solution. These new points only evaluate infeasibilities and they do not interfere with the PEM calculation process. The new points are: i) all random variables at their minimum values and ii) all random variables at their maximum values.

The two new points considered evaluate the solution's infeasibilites and they are very important in the optimization process. The PEM always evaluates the probabilistic values of a specific random variable $p_{l, k}$ fixing the values of the other random variables at their corresponding mean values $\left(\mu_{p 1}, \mu_{p 2}, \ldots, p_{l, k}, \ldots \mu_{p m}\right)$ [23], [25]. Thus, some cases are not considered in the probabilistic evaluation, which could cause a violation of the constraints.

\section{CASE Study}

The case study is the well-known system used in [6] and presented in Fig. 3. Voltage operation is $12.66 \mathrm{kV}$ and the maximum active and reactive power demands are $3,802 \mathrm{~kW}$ and 2,694 kVAr, respectively. A time horizon of four years is considered and the operation costs of each year are analyzed by means of the net present value using an annual interest rate of $10 \%$. In this case $\alpha^{\text {ope }}$ value, which updates the operating costs to the net present value, is 3.17. Three load levels are taken into account and they are shown in Table I.

The buses 17, 24, 35, 41, 46, 52, 58, 63, 67, and 69, to simulate realistic conditions, are selected as candidate buses since, in real systems, DG units can be installed in just a few buses due to the proximity of primary energy sources. Two DG types are available to be installed: stochastic (type 1 - DFIG) and dispatchable (type 2 - SG). The active power limit for DG of type 1 is $732.5 \mathrm{~kW}$ due to the mechanical limitation of this type of DG. DG data are presented in Table II. In Table III wind speed stochastic data for two wind regions where the candidate buses are located are presented. These data are based on the wind data obtained from NERL (National Renewable Energy Laboratory) [28]. In the tests we use a similar power production curve of a wind turbine from [29]. Wind speed data are approximated by a Weibull PDF, as done in several papers in literature. In this paper random variables are treated as uncorrelated variables.

Due to the limits of DG penetration, the maximum number of DG units allowed to be installed in the system is two. 


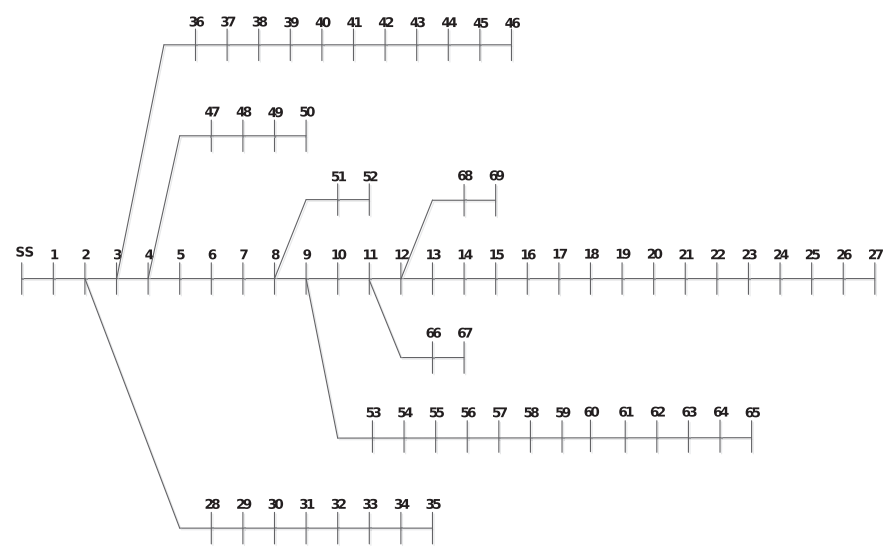

Fig. 3. Topology of 69 bus system.

TABLE I

OPERATIONAL DATA

\begin{tabular}{cccc}
\hline $\begin{array}{c}\text { Load } \\
\text { Level }\end{array}$ & $\begin{array}{c}\text { Load } \\
\text { factor }(\%)\end{array}$ & $\begin{array}{c}\text { Time } \\
\text { (h/year) }\end{array}$ & $\begin{array}{c}\text { SS energy } \\
\text { Costs }(\mathbf{\$} / \mathbf{M W h})\end{array}$ \\
\hline Light & 50 & 2000 & 31.2 \\
Nominal & 80 & 5760 & 45.6 \\
Peak & 100 & 1000 & 57.7 \\
\hline
\end{tabular}

TABLE II

DG DATA

\begin{tabular}{ccccccc}
\hline $\begin{array}{c}\text { DG } \\
\text { Type }\end{array}$ & $\begin{array}{c}\text { Inst. } \\
\text { Costs }\left(\mathbf{1 0}^{\mathbf{3}} \text { \$) }\right.\end{array}$ & $\begin{array}{c}\text { Min. } \\
\text { Ind. PF }\end{array}$ & $\begin{array}{c}\text { Min. } \\
\text { Cap. PF }\end{array}$ & $\begin{array}{c}\text { Oper. } \\
\text { Costs (\$/MVAh) }\end{array}$ & $\begin{array}{c}\text { S_min } \\
(\mathbf{k V A})\end{array}$ & $\begin{array}{c}\text { S_max } \\
(\mathbf{k V A})\end{array}$ \\
\hline $\mathbf{1}$ & 150.0 & 0.85 & 0.95 & 0.5 & 0.00 & 800.0 \\
$\mathbf{2}$ & 60.0 & 0.85 & 0.95 & 32.5 & 0.00 & 500.0 \\
\hline
\end{tabular}

Considering that the two DG units are injecting at their maximum capacities, the DG penetration levels are 40.67, 50.84 and $81.35 \%$ for the peak, nominal and light load levels, respectively.

Two switched $\mathrm{CB}$ are installed at a specific bus (300 and $600 \mathrm{kVAr}$, respectively), where $\mathrm{CB}$ power injection in this bus can be $0,300,600$ and $900 \mathrm{kVAr}$. The CB available to be placed are described in Table IV. The maximum reactive power injected by $\mathrm{CB}$ in a particular bus must be lower than 1,500 kVAr. Power factor (PF) limits are 0.93 and 1.0 (inductive) for the light and nominal load levels, and 0.93 (inductive) and 0.99 (capacitive) for the peak load level [7]. The maximum and minimum voltage limits are 1.05 and 0.95 pu, respectively.

In order to show the accuracy and efficiency of the proposed method, we have compared it with MCS. MCS is carried out with the solution provided by the proposed method, considering that 6,000 trials could represent the stochastic behavior of the problem studied and provide a "true" result of the power flow solution [25]. The statistical analysis shown in this section is based on MCS results and is carried out using the distribution fitting toolbox of MATLAB.

Three cases are analyzed in this section. In the first one, both types of DG units can be installed in the system, in the second case only stochastic DG are available to be installed, and, in the third case, only dispatchable DG can be installed.

\section{A. Both DG Types}

The solution found by the proposed method for this case presents a mean value for the investment and operational costs $(\overline{O f})$ of $\$ 3,364.88 \times 10^{3}$ and a standard deviation of $\$ 210.57 \times 10^{3}$. This solution proposes the following actions:

- switched CB allocation of $300 \mathrm{kVAr}$ at bus 64;

- fixed CB allocation of $300 \mathrm{kVAr}$ at bus 12;

- fixed CB allocation of $900 \mathrm{kVAr}$ at bus 61;

- DG installation of type 1 at bus 17 ;

- DG installation of type 2 at bus 63 .

The $(\overline{O f})$ with MCS is $\$ 3,374.04 \times 10^{3}$ with a standard deviation of $\$ 245.06 \times 10^{3}$. Therefore, considering the mean values, the proposed method's results present an error of around $0.26 \%$ compared with the MCS results. The PDF of $\overline{O f}$ is shown in Fig. 4. In Table V, the operational scheme of DG and switched CB is presented for the proposed solution. The values presented in Table $\mathrm{V}$ for the DG installed at bus 17 are the mean values. Due to the production costs, dispachable DG (bus 63 ) produces only active power for every load level and stochastic DG (bus 17), in addition to active power, produces reactive power for the nominal and peak load levels, helping CB in promoting voltage control through reactive support, loss reduction and power factor control at the substation. The PDFs for active power injected by the DG installed at bus 17 , for the peak and nominal load levels, are shown in Fig. 5. The high probability of the extreme values is due to the power production curves of the wind turbines, where $\omega_{\text {cut-in }}=4 \mathrm{~m} / \mathrm{s}$ and $\omega_{\text {rated }}=13 \mathrm{~m} / \mathrm{s}$. $\omega_{\text {cut-in }}$ and $\omega_{\text {rated }}$ are the minimum wind speed at which the wind turbine starts to produce power and the wind speed from which the wind turbine produces at its maximum active power, respectively.

The critical voltage of this system occurs at bus $65(0.90918$, 0,92873 , and 0.95670 pu for peak, nominal, and light load levels, respectively). When applying the proposed solution, this bus presents an acceptable voltage with a lower variation, 0.58 and $0.54 \%$ for the peak and nominal load levels, respectively. The stochastic DG installed at bus 17 has less influence on this bus, whereas bus 27 is directly influenced by this DG, presenting $3.55 \%$ and $3.48 \%$ of voltage variation for peak and nominal load levels, respectively. The PDFs of voltages at buses 27 and 65 for the peak load level are presented in Fig. 6. Table VI shows a comparison between mean values and standard deviations for the voltages at buses 27 and 65 by the proposed method and MCS for the peak load level. Fig. 7 shows the maximum, minimum, and mean values of the system voltage profile. DG penetration in terms of mean values represents $10.84,17.76$ and $18.89 \%$ for the light, nominal, and peak load levels, respectively. The maximum penetration occurs when both DGs produce at their maximum power, this value being $27.90 \%$.

\section{B. Only Stochastic DG}

When only stochastic DG is considered, the proposed method finds a solution with $\overline{O f}$ equal to $\$ 3,538.18 \times 10^{3}$. Applying MCS, $\bar{O} f$ is $\$ 3,528.24 \times 10^{3}$, showing the efficiency of the proposed method due to smaller errors $(0.26 \%$ for case A and $0.28 \%$ for case B). Fig. 8 shows the $\overline{O f}$ 's PDF. 
TABLE III

Stochastic Wind Speed Data

\begin{tabular}{|c|c|c|c|c|c|c|c|c|c|c|c|c|c|}
\hline Region & $\begin{array}{c}\text { Candidate } \\
\text { Buses }\end{array}$ & $\operatorname{Mean}(\mathbf{m} / \mathbf{s})$ & $\begin{array}{l}\text { Load Level } \\
\text { St. Dev }\end{array}$ & 1 Kurt. & Skew & Mean $(\mathrm{m} / \mathrm{s})$ & $\begin{array}{c}\text { Load Leve } \\
\text { St. Dev }\end{array}$ & 12 & Skew & Mean $(\mathrm{m} / \mathrm{s})$ & $\begin{array}{l}\text { Load Level } \\
\text { St. Dev }\end{array}$ & $\begin{array}{l}3 \\
\text { Kurt. }\end{array}$ & Skew \\
\hline \multirow{2}{*}{1} & \multirow{2}{*}{$\begin{array}{c}17,35,41 \\
\quad 46,52\end{array}$} & 7.72 & 4.45 & 3.74 & 0.81 & 8.09 & 4.24 & 3.32 & 0.66 & 8.48 & 3.61 & 2.83 & 0.37 \\
\hline & & \multicolumn{4}{|c|}{ Weibull param. $\alpha=2.32 ; \beta=11.10$} & \multicolumn{4}{|c|}{ Weibull param. $\alpha=2.12 ; \beta=10.30$} & \multicolumn{4}{|c|}{ Weibull param. $\alpha=2.5 ; \beta=9.00$} \\
\hline \multirow{2}{*}{2} & \multirow{2}{*}{$\begin{array}{c}24,58,63 \\
67,69\end{array}$} & 8.59 & 5.12 & 3.47 & 0.78 & 7.30 & 5.23 & 4.33 & 1.12 & 8.62 & 4.02 & 2.87 & 0.43 \\
\hline & & \multicolumn{4}{|c|}{ Weibull param. $\alpha=2.38 ; \beta=11.56$} & \multicolumn{4}{|c|}{ Weibull param. $\alpha=1.96 ; \beta=10.02$} & \multicolumn{4}{|c|}{ Weibull param. $\alpha=2.22 ; \beta=9.70$} \\
\hline
\end{tabular}

TABLE IV

CAPACITOR BANKS DATA

\begin{tabular}{cccc}
\hline Type & $\begin{array}{c}\text { Power } \\
\text { (kVAr) }\end{array}$ & $\begin{array}{c}\text { Fixed CB } \\
\text { Costs (\$) }\end{array}$ & $\begin{array}{c}\text { Switched CB } \\
\text { Costs (\$) }\end{array}$ \\
\hline $\mathbf{1}$ & 300 & 4050.0 & 6450.0 \\
$\mathbf{2}$ & 600 & 5150.0 & 7650.0 \\
$\mathbf{3}$ & 900 & 6550.0 & 9550.0 \\
$\mathbf{4}$ & 1200 & 7500.0 & 10150.0 \\
$\mathbf{5}$ & 1500 & 8075.0 & 10950.0 \\
\hline
\end{tabular}

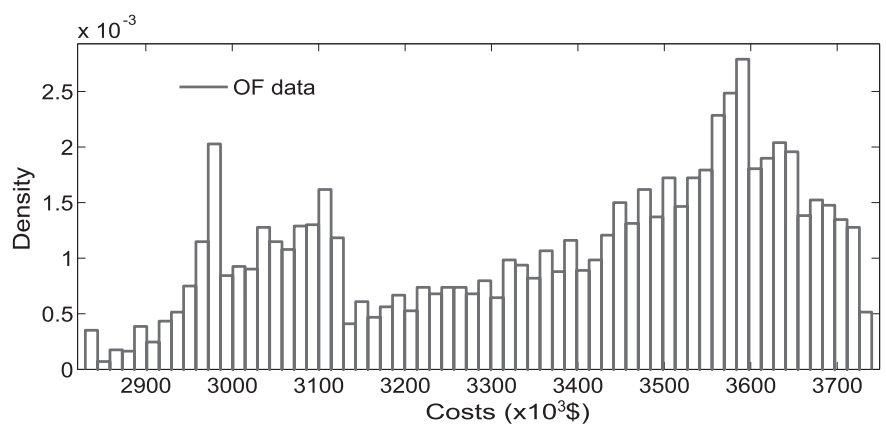

Fig. 4. Objective function PDF.

TABLE V

POWER INJECTION OF CB AND DG (KW AND KVAR)

\begin{tabular}{ccccc}
\hline & Bus & Light & Nominal & Peak \\
CB & $\mathbf{6 4}$ & $0.0+\mathrm{j} 0.0$ & $0.0+\mathrm{j} 0.0$ & $0.0+\mathrm{j} 300.0$ \\
\hline \multirow{2}{*}{ DG } & $\mathbf{1 7}$ & $221.90+\mathrm{j} 16.27$ & $296.24+\mathrm{j} 139.34$ & $337.9+\mathrm{j} 174.05$ \\
& $\mathbf{6 3}$ & $282.82+\mathrm{j} 0.0$ & $500.0+\mathrm{j} 0.0$ & $500.0+\mathrm{j} 0.0$ \\
\hline
\end{tabular}

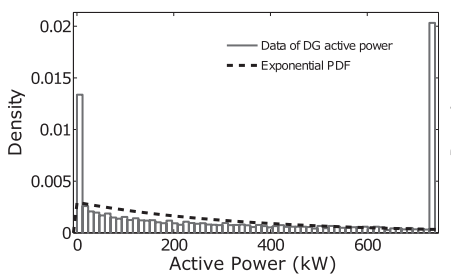

(a)

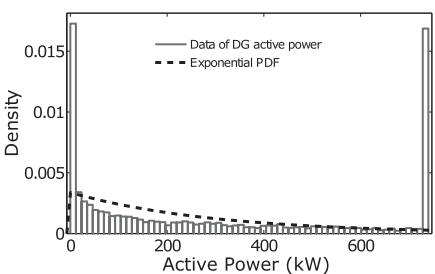

(b)
Fig. 5. Active power data by MCS for DG installed at bus 17: (a) Peak load level; (b) Nominal load level.

The solution found proposes the following actions:

- fixed CB allocation of $300 \mathrm{kVAr}$ at bus 16;

- a fixed CB allocation of $600 \mathrm{kVAr}$ at bus 61;

- a switched CB allocation of $1500 \mathrm{kVAr}$ at bus 60 ;

- a switched CB allocation of $600 \mathrm{kVAr}$ at bus 64 ;

- installation of DG unit of type 1 at bus 63 .

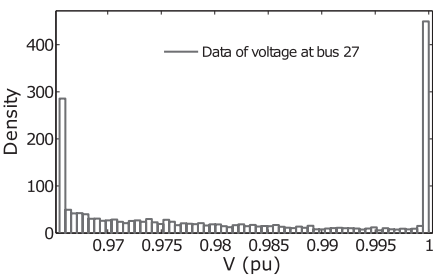

(a)

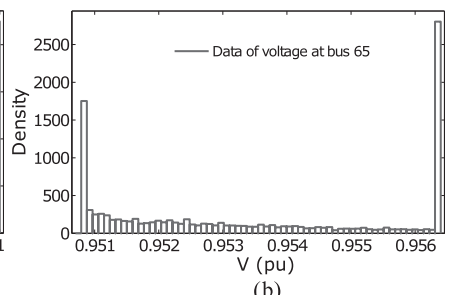

(b)
Fig. 6. Voltage data for peak load level: (a) Bus 27; (b) Bus 65.

TABLE VI

Mean Values of Voltages With MCS and PEM

\begin{tabular}{lcccc}
\hline & \multicolumn{2}{c}{$V_{27}$} & \multicolumn{2}{c}{$V_{65}$} \\
& $\mu(\mathrm{pu})$ & $\sigma$ & $\mu(\mathrm{pu})$ & $\sigma$ \\
\hline PEM & 0.9822 & 0.0129 & 0.9534 & 0.0018 \\
MCS & 0.9818 & 0.0133 & 0.9535 & 0.0021 \\
\hline
\end{tabular}

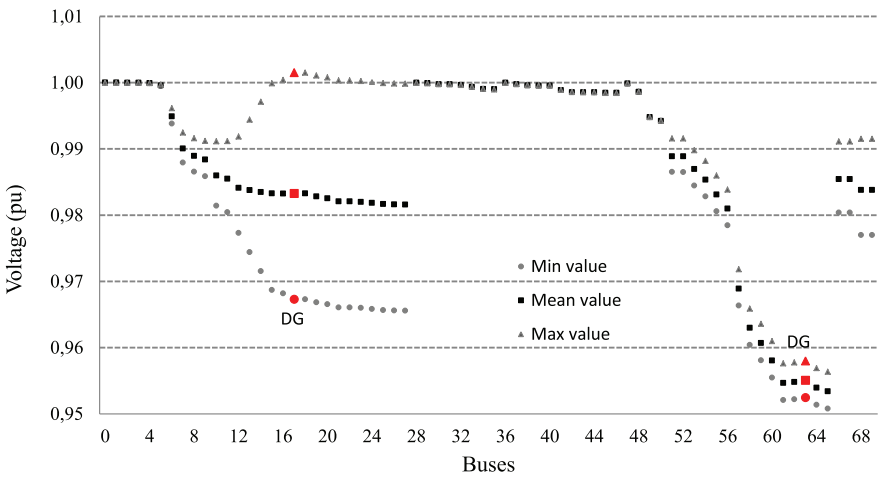

Fig. 7. Voltage profile with both DG types.

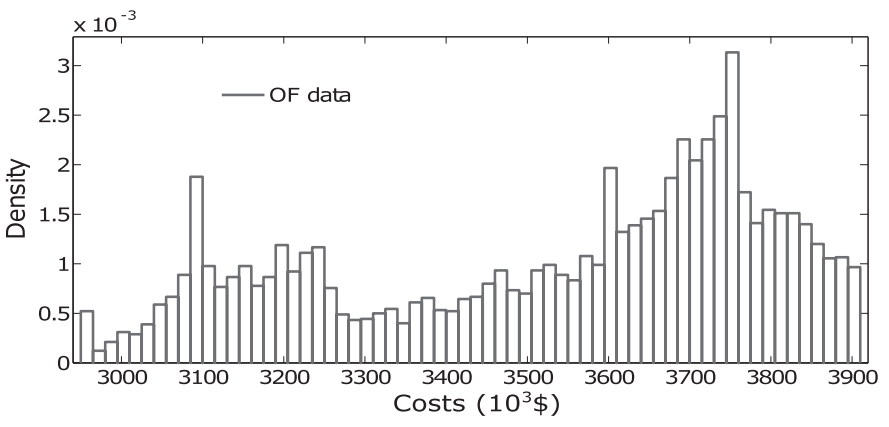

Fig. 8. Objective function PDF with only stochastic DG.

The operational scheme of the devices installed in the system is presented in Table VII. In this case, the values for DG injections are the mean values. Voltage variations at buses 27 and 65 are 0.43 and $2.65 \%$, respectively, and Table VIII shows a 
TABLE VII

POWER INJECTION OF CB AND DG (KW AND KVAR)

\begin{tabular}{crccc}
\hline & Bus & Light & Nominal & Peak \\
CB & $\mathbf{6 0}$ & $0.0+\mathrm{j} 0.0$ & $0.0+\mathrm{j} 0.0$ & $0.0+\mathrm{j} 1500.0$ \\
& $\mathbf{6 4}$ & $0.0+\mathrm{j} 0.0$ & $0.0+\mathrm{j} 600.0$ & $0.0+\mathrm{j} 600.0$ \\
\hline DG & $\mathbf{6 3}$ & $265.01+\mathrm{j} 0.0$ & $274.63-\mathrm{j} 90.27$ & $357.32-\mathrm{j} 117.40$ \\
\hline
\end{tabular}

TABLE VIII

Mean Values of Voltages With MCS and PEM

\begin{tabular}{ccccc}
\hline & \multicolumn{2}{c}{$V_{27}$} & \multicolumn{2}{c}{$V_{65}$} \\
& $\mu(\mathrm{pu})$ & $\sigma$ & $\mu(\mathrm{pu})$ & $\sigma$ \\
\hline PEM & 0.97119 & 0.00872 & 0.96196 & 0.00153 \\
MCS & 0.97111 & 0.00994 & 0.96245 & 0.00166 \\
\hline
\end{tabular}

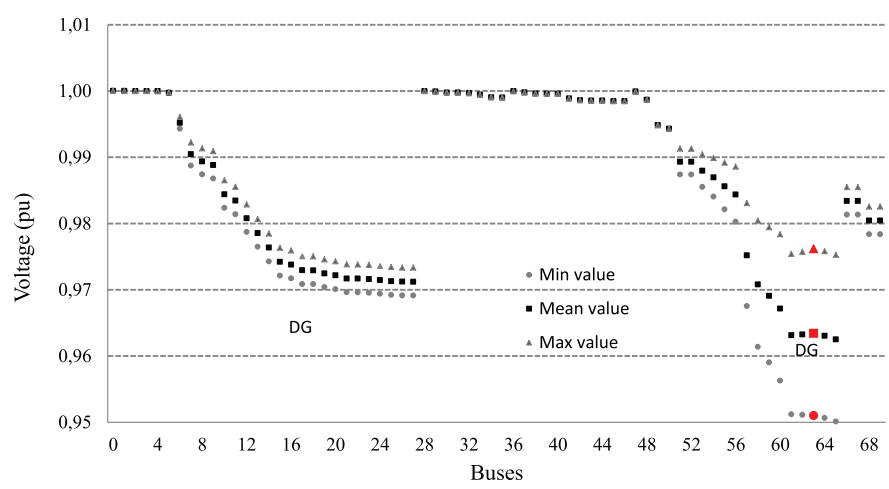

Fig. 9. Voltage profile with only stochastic DG for the peak load level.

comparison between PEM and MCS values of the voltage buses for the peak load level.

In this case, due to the greater influence of the probabilistic behavior of DG, more CB are installed in the system to ensure compliance with voltage constraints. However, in order not to violate power factor limits at the substation due to the amount of reactive power injected by $\mathrm{CB}$, the DG installed at bus 63 absorbs reactive power at the nominal and peak load levels. The voltage behavior is depicted in Fig. 9.

In order to better assess the impact of the two new points used in the OPF as mentioned in subsection III-C, we will consider as an arbitrary solution the CBs and DG positions in the solution presented in subsection IV-B by adding another stochastic DG at bus 24 . The OPF solution considering only the $2 m+1$ points provides a feasible solution, however, when this solution is evaluated by MCS it has infeasibilites for some scenarios. In Fig. 10 the cumulative probability function $(\mathrm{CDF})$ of the power factor at a substation for a nominal load level is presented.

We can observe a high probability (over 20\%) that this specific constraint is violated, when the two points are not considered. This can occur with any problem constraints during the optimization process, resulting in solutions with a high probability of violated constraints. When we use the two new points this problem is minimized or even disappears, as we can see in Fig. 10. The solutions found using the two points are generally higher than the ones without using the two points.

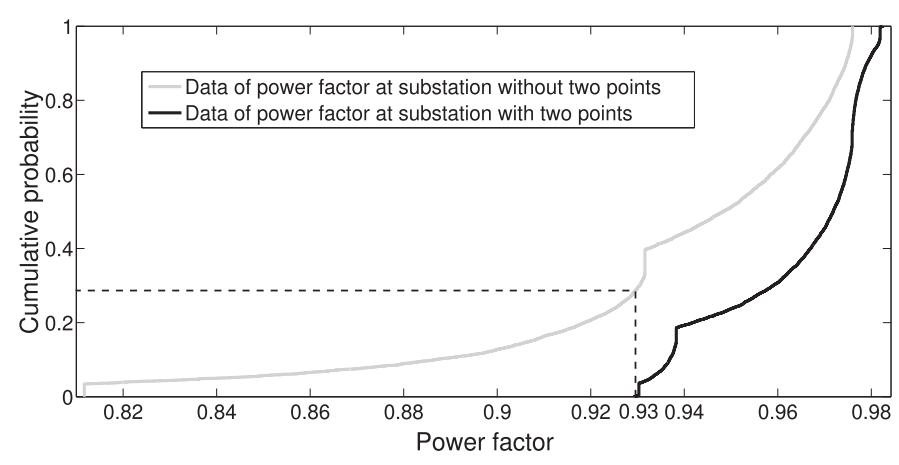

Fig. 10. Power factor at a substation.

TABLE IX

POWER INJECTION OF CB AND DG (KW AND KVAR)

\begin{tabular}{crccc}
\hline \multirow{2}{*}{ CB } & $\begin{array}{r}\text { Bus } \\
\mathbf{1 2}\end{array}$ & $\begin{array}{c}\text { Light } \\
0.0+\mathrm{j} 0.0\end{array}$ & $\begin{array}{c}\text { Nominal } \\
0.0+\mathrm{j} 600.0\end{array}$ & $\begin{array}{c}\text { Peak } \\
0.0+\mathrm{j} 600.0\end{array}$ \\
\hline \multirow{2}{*}{ DG } & $\mathbf{5 8}$ & $0.0+\mathrm{j} 0.0$ & $500.0+\mathrm{j} 0.0$ & $494.95+\mathrm{j} 35.51$ \\
& $\mathbf{6 3}$ & $323.23+\mathrm{j} 0.0$ & $500.0+\mathrm{j} 0.0$ & $494.95+\mathrm{j} 57.20$ \\
\hline
\end{tabular}

\section{Only Dispatchable DG}

With only dispatchable DG, the solution found has an $O f$ equal to $\$ 3,456.31 \times 10^{3}$. In this case the problem becomes a deterministic one, without probabilistic variables. The actions proposed are:

- switched CB allocation of $600 \mathrm{kVAr}$ at bus 12;

- a fixed CB allocation of $900 \mathrm{kVAr}$ at bus 61 ;

- installation of DG unit of type 2 at buses 58 and 63 .

The operational scheme of CB and DG for this case is presented in Table IX.

When the DGs installed are dispatchable, their power productions are constant, for this reason smaller $\mathrm{CB}$ are needed to operate within the operational limits. The critical voltage in this case is 0.9616 pu at bus 65 for the peak load level.

\section{Discussion of the Results}

It can be observed that DG locations change for each case study. Dispatchable DGs are installed at buses 63 and 58 for cases $A$ and $C$ due to their constant power production and because this region has the biggest load concentration of the system, causing a lower voltage variation in this region compared with case $B$, where the DG installed at bus 63 is stochastic. The variation caused by the stochastic nature of the DG in cases $A$ and $B$ is offset by the larger amount of reactive power injected by $\mathrm{CB}$, making the system capable of operating under any wind condition.

In case $A$ the stochastic DG installed at bus 17 provides some benefits to the system in addition to lower production costs. The benefits of this DG are not only economic, but also environmental because it uses renewable energy sources.

\section{CONCLUSIONS}

In active distribution systems the benefits and undesirable effects obtained by the installation or existence of DG and 
$\mathrm{CB}$ are directly related to the locations of these devices in the network and their operation modes. Thus, this paper presents a methodology for the simultaneous CB and DG allocation, taking into account the presence of stochastic DG.

As it can be seen in the results presented in the previous section, the system's operational state is susceptible to DG operation, causing a large voltage variation. The results presented show that the proposed methodology is very efficient in finding the buses where $\mathrm{CB}$ and DG are to be allocated, as well as the control scheme of the switched CB and DG dispatches, considering physical and operational constraints. The different results of the case studies have shown that the operation of $\mathrm{CBs}$ and DG is very dependent on the type of DG (stochastic or dispatchable) and the reactive power produced by the CBs, making the methodology proposed valuable in a wide range of cases.

These results can be used when planning the system according to the needs and interests established by the planning divisions of distribution companies.

\section{REFERENCES}

[1] W. El-Khattam, Y. Hegazy, and M. Salama, "An integrated distributed generation optimization model for distribution system planning," IEEE Trans. Power Syst., vol. 20, no. 2, pp. 1158-1165, May 2005.

[2] S. G. Naik, D. Khatod, and M. Sharma, "Optimal allocation of combined DG and capacitor for real power loss minimization in distribution networks," Int. J. Elect. Power Energy Syst., vol. 53, pp. 967-973, 2013.

[3] S. Engelhardt et al., "Reactive power capability of wind turbines based on doubly fed induction generators," IEEE Trans. Energy Convers., vol. 26, no. 1, pp. 364-372, Mar. 2011.

[4] S. Haffner, L. F. A. Pereira, L. A. Pereira, and L. S. Barreto, "Multistage model for distribution expansion planning with distributed generationPart I: Problem formulation," IEEE Trans. Power Del., vol. 23, no. 2, pp. 915-923, Apr. 2008.

[5] K. Mahmoud, N. Yorino, and A. Ahmed, "Optimal distributed generation allocation in distribution systems for loss minimization," IEEE Trans. Power Syst., 2015, doi: 10.1109/TPWRS.2015.2418333

[6] M. E. Baran and F. F. Wu, "Optimal capacitor placement on radial distribution systems," IEEE Trans. Power Del., vol. 4, no. 1, pp. 725-734, Jan. 1989.

[7] B. Milosevic and M. Begovic, "Capacitor placement for conservative voltage reduction on distribution feeders," IEEE Trans. Power Del., vol. 19, no. 3, pp. 1360-1367, Jul. 2004.

[8] C.-S. Lee, H. V. H. Ayala, and L. dos Santos Coelho, "Capacitor placement of distribution systems using particle swarm optimization approaches," Int. J. Elect. Power Energy Syst., vol. 64, pp. 839-851, 2015.

[9] T. Niknam, "A new HBMO algorithm for multiobjective daily volt/var control in distribution systems considering distributed generators," Appl. Energy, vol. 88, no. 3, pp. 778-788, 2011.

[10] B. A. de Souza, H. do Nascimento Alves, and H. A. Ferreira, "Microgenetic algorithms and fuzzy logic applied to the optimal placement of capacitor banks in distribution networks," IEEE Trans. Power Syst., vol. 19, no. 2, pp. 942-947, May 2004.

[11] T. Niknam, B. B. Firouzi, and A. Ostadi, "A new fuzzy adaptive particle swarm optimization for daily volt/var control in distribution networks considering distributed generators," Appl. Energy, vol. 87, no. 6, pp. 1919-1928, 2010.

[12] M. H. Moradi, A. Zeinalzadeh, Y. Mohammadi, and M. Abedini, "An efficient hybrid method for solving the optimal sitting and sizing problem of DG and shunt capacitor banks simultaneously based on imperialist competitive algorithm and genetic algorithm," Int. J. Elect. Power Energy Syst., vol. 54, pp. 101-111, 2014.

[13] A. Mahari and A. Mahari, "Optimal DG and capacitor allocation in distribution systems using DICA," J. Eng. Sci. Technol., vol. 9, no. 5, pp. 641-656, 2014.

[14] O. Ausavanop, A. Chanhome, and S. Chaitusaney, "An optimal allocation of distributed generation and voltage control devices for voltage regulation considering renewable energy uncertainty," IEEJ Trans. Elect. Electron. Eng., vol. 9, no. S1, pp. S17-S27, 2014.
[15] A. Ahmidi, X. Guillaud, Y. Besanger, and R. Blanc, "A multilevel approach for optimal participating of wind farms at reactive power balancing in transmission power system," IEEE Syst. J., vol. 6, no. 2, pp. 260-269, Jun. 2012.

[16] R. A. Gallego, A. J. Monticelli, and R. Romero, "Optimal capacitor placement in radial distribution networks," IEEE Trans. Power Syst., vol. 16, no. 4, pp. 630-637, Nov. 2001.

[17] G. Muñoz-Delgado, J. Contreras, and J. M. Arroyo, "Joint expansion planning of distributed generation and distribution networks," IEEE Trans. Power Syst., vol. 30, no. 5, pp. 2579-2590, Sep. 2015.

[18] S. Montoya-Bueno, J. I. Muñoz, and J. Contreras, "A stochastic investment model for renewable generation in distribution systems," IEEE Trans. Sustain. Energy, vol. 6, no. 4, pp. 1466-1474, Oct. 2015.

[19] EDSO. (2015). Flexibility: The Role of DSOs in Tomorrow's Electricity Market [Online]. Available: http://www.edsoforsmartgrids.eu/wpcontent/uploads/public/EDSO-views-on-Flexibility-FINAL-May-5th2014.pdf

[20] F. Glover. (1996). Tabu Search Fundamentals and Uses, Citeseer [Online]. Available: http://leeds-faculty.colorado.edu/glover/TS/\%20$1 \% 20$ Fundamentals $/ \% 26$ Uses.pdf

[21] P. C. Chu and J. E. Beasley, "A genetic algorithm for the generalised assignment problem," Comput. Oper. Res., vol. 24, no. 1, pp. 17-23, 1997.

[22] B. R. Pereira Jr., A. M. Cossi, and J. R. Mantovani, "Multiobjective shortterm planning of electric power distribution systems using NSGA-II," $J$. Control Autom. Elect. Syst., vol. 24, no. 3, pp. 286-299, 2013.

[23] J. M. Morales and J. Pérez-Ruiz, "Point estimate schemes to solve the probabilistic power flow," IEEE Trans. Power Syst., vol. 22, no. 4, pp. 1594-1601, Nov. 2007.

[24] A. Soroudi, M. Ehsan, R. Caire, and N. Hadjsaid, "Possibilistic evaluation of distributed generations impacts on distribution networks," IEEE Trans. Power Syst., vol. 26, no. 4, pp. 2293-2301, Nov. 2011.

[25] C.-L. Su, "Probabilistic load-flow computation using point estimate method," IEEE Trans. Power Syst., vol. 20, no. 4, pp. 1843-1851, Nov. 2005.

[26] D. Shirmohammadi, H. Hong, A. Semlyen, and G. Luo, "A compensation-based power flow method for weakly meshed distribution and transmission networks," IEEE Trans. Power Syst., vol. 3, no. 2, pp. 753-762, May 1988.

[27] A. G. Bakirtzis, P. N. Biskas, C. E. Zoumas, and V. Petridis, "Optimal power flow by enhanced genetic algorithm," IEEE Trans. Power Syst., vol. 17, no. 2, pp. 229-236, May 2002.

[28] NERL. (2015). Brazil: Energy Resources, Wind Potential [Online]. Available: http://en.openei.org/datasets/dataset/wind-resources-by-classand-country-at-50m

[29] M. H. Ahmed, K. Bhattacharya, and M. Salama, "Analysis of uncertainty model to incorporate wind penetration in LMP-based energy markets," in Proc. 2nd Int. Conf. Elect. Power Energy Convers. Syst. (EPECS), 2011, pp. 1-8.

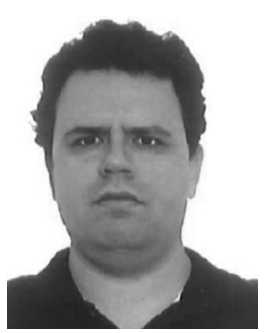

Benvindo Rodrigues Pereira Jr. received the B.Eng., M.Sc., and Ph.D. degrees in electrical engineering from the São Paulo State University (UNESP), Ilha Solteira, Brazil, in 2007, 2009, and 2014, respectively. He is a Postdoc Researcher with the Electrical Engineering and Computational Department, University of São Paulo, São Carlos, Brazil. His research interests include the planning and control of electric power systems.

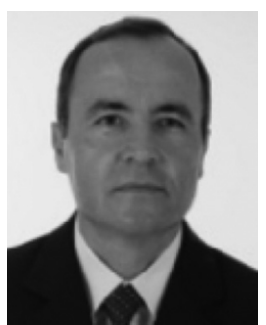

Geraldo Roberto Martins da Costa (M'88) received the B.Eng. and M.Sc. degrees in electrical engineering from São Carlos Engineering School, University of São Paulo (USP), São Paulo, Brazil, and the Ph.D. degree from the University of Campinas, Campinas, Brazil. Currently, he is a Full Professor of Electrical Engineering with São Carlos School of Engineering, USP. His research interests include power system operation and planning, particularly in the application and development of optimization methods to power systems. 


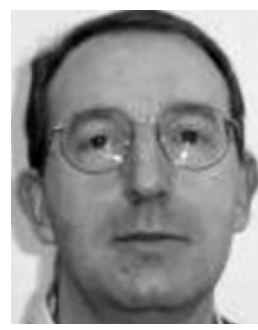

Javier Contreras (SM'05-F'15) received the B.S. degree in electrical engineering from the University of Zaragoza, Zaragoza, Spain, the M.Sc. degree from the University of Southern California, Los Angeles, $\mathrm{CA}$, USA, and the Ph.D. degree from the University of California, Berkeley, Berkeley, CA, USA, in 1989, 1992, and 1997, respectively. He is currently a Full Professor with the University of Castilla-La Mancha, Ciudad Real, Spain. His research interests include power systems planning, operations and economics, and electricity markets.

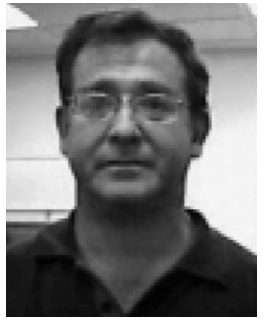

electric power systems
José Roberto Sanches Mantovani (M'06) received the degree in electrical engineering from the São Paulo State University (UNESP), Ilha Solteira, Brazil, and the Ms.C. and Ph.D. degrees in electrical engineering from the University of Campinas, São Paulo, Brazil, in 1981, 1987, and 1995, respectively. Currently, he is currently a Full Professor of Electrical Engineering with UNESP and joined the LaPSEE (Laboratorio de Planejamento de Sistemas de Energia Eletrica), Ilha Solteira, Brazil. His research interests include planning and control of 